\title{
Experience of Profiling Curricula on Cloud Computing Technologies and Engineering for Different Target Groups
}

\author{
${ }^{1}$ Yuri Demchenko, ${ }^{1}$ Adam Belloum, ${ }^{2}$ David Bernstein, ${ }^{1}$ Cees de Laat \\ ${ }^{1}$ University of Amsterdam \\ System and Network Engineering Group \\ Amsterdam, The Netherlands \\ e-mail: \{y.demchenko, a.s.z.belloum,delaat\}@uva.nl \\ ${ }^{2}$ Cloud Strategy Partners, LLC \\ Silicon Valley, California, USA \\ e-mail: david@cloudstrategypartners.com
}

\begin{abstract}
This paper presents results and experience by the authors based on the few delivered courses on Cloud Computing for different target groups of students, specialists and trainees. The developed courses implement the proposed by the authors instructional methodology integrating the two major concepts of effective learning: the Bloom's Taxonomy of cognitive learning processes and Andragogy as the adult learning methodology. The central part of proposed approach is the Common Body of Knowledge in Cloud Computing (CBK-CC) that defines the professional level of knowledge in the selected domain and allows consistent curricula structuring and profiling. The paper presents the structure of the courses and explains the principles used for developing course materials, such as Bloom's Taxonomy applied for technical education, and andragogy instructional model for professional education and training. The developed course are based on the well-defined Cloud Computing architecture, service and operational model, and stakeholder roles/responsibilities. The paper provides a short description of the developed education and training courses on Cloud Computing that illustrate how the proposed CBK-CC and instructional methodologies are used in different learning environments and for different learners' groups.
\end{abstract}

Keywords- Education and Training on Cloud Computing Technologies; Instructional methodology; Common Body of Knowledge; Bloom's Taxonomy; Andragogy; Cloud Services Model; Intercloud Architcture Framework.

\section{INTRODUCTION}

Cloud Computing technologies [1, 2] are widely used by both industry and research community; clouds provide a powerful platform for enterprise IT infrastructure migration and new applications and services development. Cloud Computing incorporates advances in computing, networking, storage technologies and provides a basis for new technology development such as Big Data and pushing further mobility based pervasive computing concept.

Recent developments in clouds are featured by growing volume of business oriented cloud applications and general trend of moving traditional packaged applications (such as business management software) to cloud services provided on the subscription basis. This allows making important business applications affordable for Small and Medium Businesses (SMB). At the same time, cloud based applications change the way how the companies organise and manage their IT infrastructure and services. Besides changing relations between capex and opex, the cloud based services makes companies more agile and responsive to new technologies and business models, in particular data driven.

This motivates a growing demand for specialists with strong technical background and deep knowledge of the Cloud Computing technologies. However, there is no widely available Cloud Computing training and professional education as well as there is no common approach to building professional level Cloud Computing curricula.

The presented paper extends and updates previously published paper by the authors [3] with the new results and experience. The curriculum design approach outlined here presents a basis for building complete set of courses in Cloud Computing, aimed at the cloud/IT architects, project managers, technical product planners, consultants, or engineers, as well as using different educational media such as face to face lectures/teaching, online education, and selftraining.

The remainder of the paper is organized as follows. Section II explains requirements to the professional education on Cloud Computing and outlines the main properties of the professional level of knowledge. Section III briefly refers to the main Cloud Computing concepts and architecture, including stakeholder and operational roles definition, used for building consistent Cloud Computing curricula. Section IV introduces the Common Body of Knowledge in Cloud Computing (CBK-CC) and explains inter-relations between its elements. Section $\mathrm{V}$ provides an example of mapping between CBK-CC components and stakeholder domains. Section VI provides a short summary of the Bloom's Taxonomy and Andragogy as key concepts used in the proposed instructional model. Section VII provides information about three educational courses developed for different learners groups. Section VIII provides summary and authors' plan for further work.

II. DEMAND FOR PROFESSIONAL EDUCATION AND TRANING ON CLOUD COMPUTING

Based on the authors' experience in delivering educational and training courses on different computer and network related topics to different learner audiences we identified a number of specifics in developing professional education and training on Cloud Computing as a new evolving technology.

The discussed here curriculum seeks to provide knowledge both in Cloud Computing and the underlying technologies. Our objective is to empower future professionals with the ability to develop new knowledge and build stronger expertise in Cloud, and also to prepare a basis for new emerging technologies such as Big Data.

The professional education needs to empower the future specialists with the following level of knowledge:

1) Knowing basic concepts and major application areas

2) Knowing similar concepts (and concepts inter-relation) and alternatives, as well as application specific areas

3) Knowing basic technologies and their relation to basic concepts. 
Cloud computing is still a new technology, but it is becoming a common preferred platform for all current and future developments. Professionals want to become an "expert" in this new area. They need both a general professional knowledge and understanding of the main development areas, as well as practical experience with activities such as projects, writing technical documents, and following and contributing to standardization.

As the result of the proposed curriculum, professionals should become "cloud aware", armed with "cloud powered design" and capable of "cloud powered analysis and thinking”.

\section{ClOUd COMPUTING FOUNDATION AND CONCEPTS}

The key component of the consistent course organization is the strong conceptual and architectural background used for creating logical structure between topics/modules, learning targets and their relation to practical business and operational needs. Well defined architectural background provides a basis for consistent vocabulary and stakeholder roles, actions and interest definition.

\section{A. Cloud Service Model and Intercloud Architecture Framework}

The effective course model should be based on the strong conceptual and architectural basis. The proposed courses are using the key industry standards on Cloud Computing such as developed by NIST [1, 2] and ITU-T [4] and Intercloud Architecture Framework (ICAF) [5, 6] proposed by authors that includes such components as the multi-layer Cloud Services Model (CSM), Intercloud Federation Framework (ICFF), Intercloud Management and Operation Framework (ICOMF), Intercloud Security Framework (ICSF) that are compliant with the recent development by the standard organisations and reflect current trends for heterogeneous intercloud services models and cloud based application integration.

The multi-layer CSM [5] reflects the main functional layers and related functional components in a typical cloud infrastructure. It combines the basic cloud service models IaaS, PaaS, SaaS into one Cloud Services Layer that typically expose standard based interfaces to user applications or services but can use proprietary interfaces to the underlying provider platform. The CSM includes also the virtualization and cloud management layers and user applications layer. The CSM providers a good model for mapping the required knowledge and skills to different roles and stakeholders in Cloud Computing.

\section{B. Main Stakeholders and Operational Roles}

To be effective and market successful, education and training program should target the main stakeholder groups and their interests in the cloud services delivery model.

We define the following main actors and roles extending the NIST stakeholder roles [2, 7]:

- Cloud Service Provider (CSP) as an entity providing cloud based services to customers, on their request and based on the Service Level Agreement (SLA).

- Cloud Broker is an entity that may play a role of the third party in offering cloud service adding value of negotiating with many CSPs or customer groups.

- Cloud Service Operator is an entity that provides a value added service of integrating services from multiple cloud providers, delivering them to the customer and operating the resulting infrastructure.
- Cloud Service Integrator is a third party IT company to design and deploy the cloud services infrastructure that matches the customer requirements.

- Cloud Auditor is a party that can conduct independent assessment of cloud services operations, performance and security of the cloud implementation.

- Cloud Carrier is an intermediary that provides connectivity and transport of cloud services from Cloud Providers to Cloud Consumers.

- Customer (like enterprise or university) is an entity that requests service from and has contractual relations with the CSP; customer does cloud services management themselves or outsource it to the third party.

- User is an end-user consuming cloud based services; in cloud services provisioning.

Typical professional profiles includes the following roles/positions (that may apply for both cloud provider and cloud customer):

- Cloud Architect

- Cloud Developer

- Cloud Applications Developer

- Cloud Administrator

- Cloud/IT Security Manager/Administrator

- Cloud/IT Infrastructure Manager

Addressing education and training skills requirements for the major stakeholders and operational roles is a condition for the course acceptance by the industry and wide professional community.

\section{COMMON Body OF KNOWLEdGE IN ClOUd COMPUTING (CBK-CC)}

CBK refers to several domains or operational categories into which Cloud Computing theory and practices breaks down. It is like a Blueprint or Framework which students can see how things fit together. For Cloud Computing, we identify the following CBK elements:

1. Cloud Computing Architectures, Intercloud Architecture, service and deployment models, stakeholder model

2. Cloud Computing platforms, software and API's

3. Cloud Services Engineering and Applications design, Design for Multi-tenancy

4. Virtualization technologies (Compute, Storage, Network)

5. Computer Networks, Software Defined Networks (SDN), Cloud/Intercloud Services Delivery Infrastructure

6. Service Computing, Web Services and Service Oriented Architecture (SOA), Mobile Cloud Computing

7. Computing models: Grid, Distributed, Cluster Computing, Scalable and Massive Parallel Computing

8. Security Architecture and Models, Operational Security, eDiscovery, Data Protection, Privacy

9. IT Service Management, Business Continuity Planning

10. Business and Operational Models, Cloud Economics, Compliance, Certification, Assurance, Dependability

Figure 1 shows the 2D relations between the CBK components and illustrates their mapping to the required skills and course subsets or profiles. Accordingly, the core Cloud Computing Fundamentals course includes such topics as Cloud Computing Architectures, service and deployment models; Cloud Computing platforms, software/middleware and API's; Cloud aware Services Design; Compute and Storage Virtualization; Web Services and Service Oriented Architecture (SOA); Cloud Security, Operational Security, Identity management; Business and Operational Models, Compliance, Assurance. 
The Cloud Computing Fundamentals provide sufficient basis for performing the main professional tasks and further independent professional development. Different groups of the cloud professionals and specialists can extend their education and training with the specific knowledge and skills according to the CBK-CC.

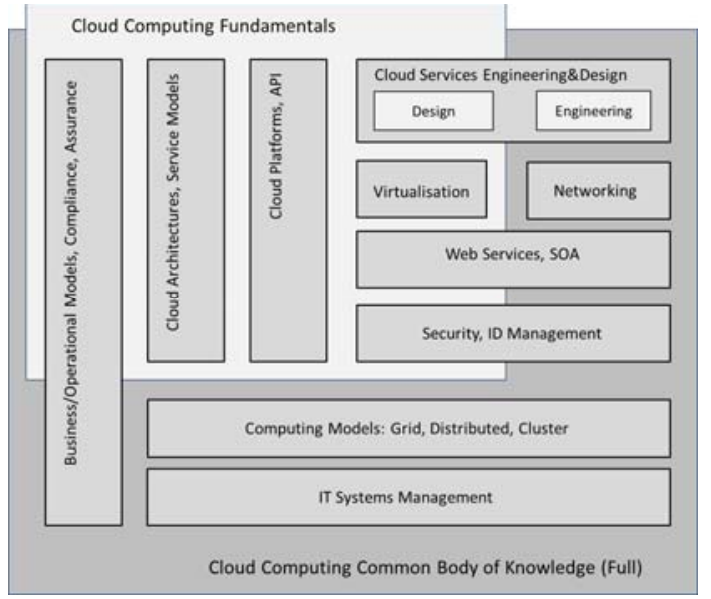

Figure 1. CBK-CC elements inter-relation and mapping to course profiles (i.e. Cloud Computing fundamentals and full course).

\section{MAPPING STAKEHOLDER DOMAINS AND COURSE COMPONENTS}

Figure 2 illustrates mapping between CSM, major stakeholder service domains, and required knowledge (or competencies). It is an important next step in building consistent curricula. This is also important for effective course profiling for the target group of students or trainees that can be used for defining the course modules that will be delivered to different groups of attendees/subscribers/listeners.

We understand the difference for students where we can just deliver a complete program, and an advance professional training where the educator needs to more specifically target the specific interests of trainees.

The following major course modules can be defined:

- Cloud Service Platforms module targets both cloud services developers (using available cloud services platforms) and technical personnel of the cloud service providers which both require deeper knowledge of the cloud services design, software, virtualization platforms, operations and business processes. It is understood that the future employees will require special training by their employer company, but the course will provide all the basic knowledge for their quick mastering of the provider or enterprise specific technologies.

- Cloud/Intercloud Services Integration, Hybrid Clouds module targets wide range of businesses (including cloud service integrators, operators, and also cloud carriers) that provide cloud integration services for enterprises and large cooperative projects. This module includes detailed analysis of the customer requirements, workflow and current IT infrastructure study and proposing the optimized cloud based infrastructure.

- Cloud Enterprise and User Applications module targets a wide group of the cloud users that need to use cloud services provided by the public CSP or private clouds run by enterprise. This user group should have also skills to develop their own cloud applications using one of the available cloud development and management platforms in the private or public cloud. This module will be also recommended for non-IT companies IT staff to be able to efficiently support internal users.

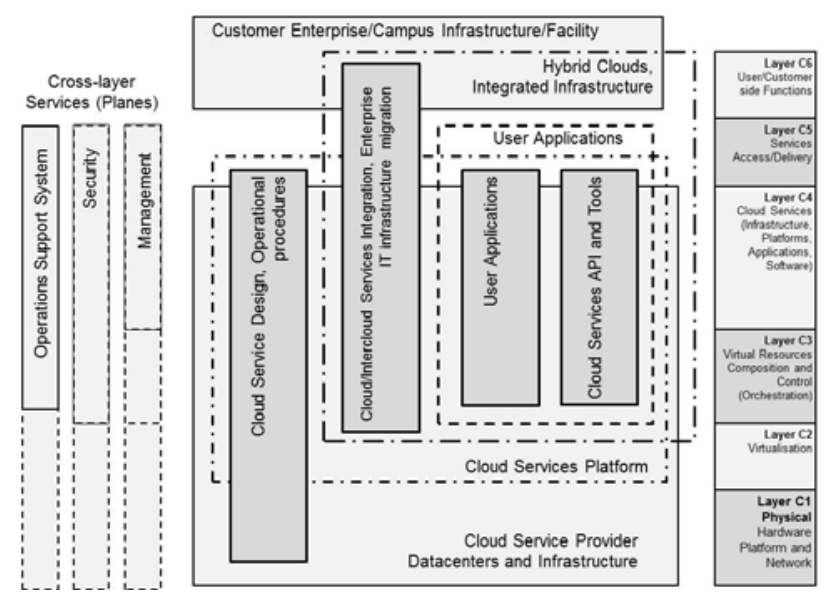

Figure 2. Mapping between Cloud Services Model (CSM), major stakeholder service domains, and required knowledge/skills.

The courses may also be profiled for different learner and professional groups (full time students, vocational or online education) and different delivery forms (lectures and labs, self-training courses, online classrooms, MOOC), example of which are discussed in the implementation section.

\section{USING BLOOM'S TAXONOMY AND ANDRAGOGY FOR DEFINING LEARNING TARGETS AND OUTCOME}

\section{A. Bloom's Taxonomy Summary and Example}

Bloom's Taxonomy is a basis for defining learning targets and modules outcome $[8,9,10]$. It sequences learning into a number of cognitive levels defining learning outcome:

\section{Knowledge}

Exhibit memory of previously learned materials by recalling facts, terms, basic concepts and answers

\section{Comprehension}

Demonstrate understanding of facts and ideas by organizing, comparing, translating, interpreting, describing, and stating the main ideas

\section{Application}

Using new knowledge, solve problems in new situations by applying acquired knowledge, facts, techniques and rules in a different way

\section{Analysis}

Examine and break information into parts by identifying motives or causes. Make inferences and find evidence to support generalizations

\section{Synthesis}

Compile information together in a different way by combining elements in a new pattern or proposing alternative solutions

\section{Evaluation}

Present and defend opinions by making judgments about information, validity of ideas or quality of work based on a set of criteria

Refer to the previous authors work [3] for the suggested mapping between Bloom's Taxonomy and required professional activity knowledge/skills and for discussion about using Bloom's Taxonomy for constructing discussion and test questions to facilitate the different cognitive learning levels and

\section{B. Methodology of Andragogy (vs. Pedagogy)}

Andragogy consists of learning strategies focused on adults $[11,12,13]$. It is often interpreted as the process of 
engaging adult learners with the structure of learning experience. The andragogy principles are highly related to professional education that typically has high motivation factor. It can be applied in on-campus and on-line education. It was developed by American educator Malcolm Knowles [20] and comprises of the six assumptions related to motivation of adult learning:

1. Adults need to know the reason for learning something (Need to Know)

2. Experience (including error) provides the basis for learning activities (Foundation)

3. Adults need to be responsible for their decisions on education; involvement in the planning and evaluation of their instruction (Self-concept)

4. Adults are most interested in learning subjects having immediate relevance to their work and/or personal lives (Readiness).

5. Adult learning is problem-centered rather than contentoriented (Orientation)

6. Adults respond better to internal versus external motivators (Motivation)

Andragogy provides effective approach to online higher education as described below.

\section{IMPLEMENTATION EXAMPLES AND LESSONS LEARNT}

This section will discuss available experience by the authors in implementing Cloud Computing courses for different target groups undertaken by different universities and communities.

\section{A. Web Services and Cloud Computing Course at the University of Amsterdam}

\section{1) Course organisation}

The Web Services and Cloud Computing course has been developed for the Master Computer Science full-time students and counts for 6 credits [14]. The course runs 2 months and has 8 contact hours per week of which 4 hours are lectures and 2 hands-on lab slots of two hours each. The course also includes the final project discussed below.

The course is built in such a way that to facilitate the students deeper study/research into the subject of Cloud Computing and related problems, improve their skill in working with professional and scientific literature, facilitate collaborative learning and actively use their knowledge in the discussion.

To achieve this goal the course employs two types of selfstudy activities: (1) discussion of the selected papers in the classroom (also called "reading club") that is done weekly and requires submitting two short summaries of the paper before discussion and after discussion where the student is required demonstrate their understanding of the paper and its relation to the weekly lecture topic; and (2) literature based research on individually selected topics that must be resulted in a short essay and its presentation during the special seminar session that takes place either at the end of course or discussion of the selected papers in the classroom. The proposed list of research topics includes more than 30 topics covering all aspects of Cloud Computing and cloud services design; each topic is provided with the initial list of 2-3 references and the students are required to find other few authoritative references.

The course will use periodical (weekly or by group of topics) knowledge testing with the test developed to assess at least first three Bloom's taxonomy cognitive levels: evaluation, synthesis and analysis, that will employ both multiple choice questions and questions requiring textual answer. The literature research and final project are the key components to achieve the higher level of knowledge in the Cloud Computing.

Important part of the university education is developing research skills that include formulating a research problem, studying and critically evaluating scientific and technical literature. This is done via cooperation with the research staff of the department. The course also invites a few guest lectures that are given by cooperating industry partners and experts.

2) Course content

The course is organized by weekly topics that include 2 lectures, labs and selected papers discussion.

Week 1: Service Oriented Architecture and Web Services Week 2: Introduction to Cloud basic technologies; Virtualization from Grid to Cloud; Virtualization techniques Week 3: Cloud Service models: IaaS/PaaS/SaaS

Week 4: Cloud Software: OpenStack, OpenNebula, Eucalyptus, Nimbus

Week 5: Cloud service providers and offered services: AWS, Microsoft Azure, Google Apps Engine

Week 6: Cloud economics; Cloud powered services design; Cloud Security

Week 7: Guest lectures (by local researchers)

Week 8: Students presentations and exam week

\section{3) Hands-on labs and final project}

The hands-on labs paly important role in the practical mastering the cloud technologies. The course also includes the final projects to design cloud based application or service to support a selected business process or scientific workflow using one of the available cloud platforms: local cloud testbed, Amazon AWS, or Microsoft Azure. The lab assignments include the basic hands-on tasks to learn how to work with virtualisation and cloud platforms both provided by the local cloud testbed and two major cloud platforms by Amazon AWS and Microsoft Azure, what together provides a basis for the practical project realization.

The final project builds upon all the hand-on performed in the labs where students learn how to contextualize Virtual Machines, deploy simple service in VMs and stage them on various cloud resources in both private and public clouds. This simple exercise teaches the student the idea of scaling up and down on demand. In this phase student have access to limited resource on the private cloud and rapidly see that the simple services deployed in the private cloud are overloaded and thus need to create more instance which is not possible on the private cloud and thus need to scale out using public cloud. The will also experience to various application loads to learn the how to adapt their resources to the actual application loads which will involve scale down the number of VMs when needed. At this stage we do not consider cost of the VMs when scaling out, the only concern is to keep a service running at a given QoS.

When the students master the techniques and methods involved in using various platform, they will repeat the same experiments but this time they are assigned a budget which will also limit the resource they can use on the public cloud. In these experiments the students will have to pay more attention to the billing model of the cloud provides, and rethink their strategy of load balancing the work over the various VM to optimize their usage. 
When students have completed these two steps, they can start with the final project which consists in deploying application composed a set of dependent tasks (workflow) across the available resources (private and public). In term of setting the project will use all bits developed during the previous experience, the only new parameter they students have to consider is the dependency introduced at the application level.

The labs and project form a gradual introduction to cloud technology in terms of complexity. In each phase students are introduced to a new aspect of cloud system complicating more their assignment. At the end they will have built both technical skills and a mindset which prepare them real life usage of cloud technology.

\section{B. Cloud Computing Engineering course at Laureate Online Education}

\section{1) Course organisation}

The Laureate Cloud Engineering online Andragogy provides effective approach to online higher education that is offered by high ranked online universities such as Laureate Online Higher Education (LOHE) [15, 16], the online education branch of the Liverpool University. The LOHE provides fully online teaching/education environment based on customized Blackboard platform. The following activities and education forms are used in Computer Science online master education:

- The module consists of 8 weekly seminars that includes 2 Discussion Questions (DQ), Hands-in Assignment (HA, or homework) and project assignment.

- Each seminar is provided with the Lecture Notes and textbook reading assignment. There is no recorded or synchronous lectures, what makes also possible education delivery to countries and to students with low Internet connectivity.

- Discussion questions and asynchronous discussion are the main form of educational activity. DQ answers are submitted to the discussion forum and the students are required to contribute to the discussion. The students benefit from the knowledge and experience sharing during discussion and learn how to defend own answer. Instructor plays a role of moderator and the students' knowledge and activities assessor.

- Discussion questions are designed in such a way that to stimulate the students higher cognitive activities starting from the basic literature search to analyzing and evaluating collected information and further to making concise knowledge synthesis and their application to problem solving.

- Criteria for assessing the students' knowledge and learning outcome are based on the Bloom's Taxonomy and require some experience from the instructor.

2) Course content

The weekly seminars has the following topics:

Seminar 1: Cloud Computing foundation

Cloud Computing definition, reference architecture, Cloud stakeholders and roles. Cloud properties, cloud benefits and cloud challenges. General use cases analysis.

Seminar 2: Cloud architectures and cloud platforms

Cloud enabling technologies: virtualization platforms. Cloud related standards; Topology and Orchestration Specification for Cloud Applications (TOSCA), Open Cloud Computing Interface (OCCI); lower layer standards: OVF by DMTF; CDMI by SNIA.

\section{Seminar 3: Cloud Infrastructure as a Service (IaaS)}

IaaS architectures and models; cloud management functions and API; security groups, virtual machine images. Storage file system types: persistent object storage and block storage. Storage and network virtualization details. Example Amazon AWS IaaS cloud architecture and components.

\section{Seminar 4: Cloud Platform as a Service (PaaS)}

PaaS architecture components: code containers, queues, data structures, databases, load balancers. PaaS services composition and management: Azure Service Bus; Other PaaS platforms: VMware Cloud Foundry, RedShift.

Seminar 5: Cloud Software as a Service (SaaS) and Mobile clouds

Overview SaaS and application clouds: components, services and usage models. Overview and analysis of Google Apps and Office365. Mobile Cloud Computing.

Seminar 6: Cloud Security, Data protection and Privacy, Access Control and Identity Management

Cloud security and privacy challenges; existing architectures, solutions and methods; access control and security policies, Identity Management in clouds. Data security in clouds. Privacy protection in clouds. Overview security models by major cloud providers Amazon AWS and Microsoft Azure.

Seminar 7: Cloud powered services and applications design, multitenant applications

Example of using Amazon Web Services cloud to gradually design evolving company's IT infrastructure. Multitenant applications design with Microsoft Azure cloud.

Seminar 8: Cloud economics, cloud compliance and assurance

Cloud economics, cloud compliance, existing standards and industry practices, cloud dependability. Example PCI DSS compliance for clouds.

\section{Advanced on-line training course for professional IT specialists}

The authors are currently involved into ongoing implementations of the proposed principles and methodologies in the extended self-training course on Cloud Computing for IT professionals as a part of the IEEE eLearning Library program that provides wide spectrum of online educational materials for IEEE members.

1) Course organization and approach

When applying discussed above techniques, in particular andragogy and Bloom's Taxonomy to online self-education and training, the following specifics will apply:

- $\quad$ Less time to be devoted by trainee; estimated 1 hour per lesson, maximum 3 lessons per course

- $\quad$ Requires the course workflow to be maximum automated, especially if coupled with certification or pre-certification

- Requires guided activities while allowing self-directed course selection

- Provide course consistency in sense of style, presentation/graphics, etc.

- Combined with the Bloom's taxonomy, provide understood targets and outcomes

- Knowledge control questionnaires at the end of lessons or topics.

The course must be designed in such a way that to build a consistent/advanced knowledge foundation and to prepare the listeners for a concise and advanced use of the cloud technologies and tools for emerging technologies such as Big Data, data analytics and new emerging business paradigms motivated by increasing use of the smart mobile devices. 
The curriculum design must be more specifically aimed at the main stakeholders to reflect typical roles or functions such as technical product planner, consultant, or engineer. The modules must be structured along the lines of common industry terms and features of Cloud Computing, so that the student with a passing knowledge of the subject will be able to instantly recognize the subject matter of the course.

\section{2) Course content}

The online curriculum should have a pre-requisite of having basic or common knowledge in Cloud Computing and provide materials at Intermediate, Advanced, and Expert levels. The Intermediate and Advanced lessons constitute the Cloud Computing fundamentals and aimed for IT decision makers and informed users. "Advanced" parts are intended for engineers/practitioners developing and operating cloud services, and doing integration/consulting work.

The online course materials must be developed with high granularity to allow easy course composition for the target groups of trainees and also designing individual profiles.

The total number of self-paced tutorials reaches 33 tutorials that cover the following groups of topics:

Topics area 1: Cloud Computing Baseline, service models, use cases

Topics area 2: Cloud taxonomy, standardization, cloud economy

Topics area 3: Cloud architecture and platforms

Topics area 4: Cloud Infrastructure as a Service (IaaS), virtualization in cloud, AWS, cloud powered design Topics area 5: Cloud Platform as a Service (PaaS), architecture, platforms, SQL and NoSQL databases

Topics area 6: Cloud Software as a Service (SaaS), Business SaaS applications, Big Data and HPC cloud.

Topics area 7: Cloud Security, Cloud Privacy, Cloud compliance

Topics area 8: Cloud construction and cloud services delivery infrastructure

Topics area 9: Intercloud, interoperability and trends, Mobile Cloud computing.

\section{3) Supplementary components}

The training materials suite features also advanced glossary of Cloud Computing related terms and self-test questions. The self-test questions are provided after each tutorial in a form of multiple choice questions. The questions are designed in such a way that to initiate higher cognitive learning levels according to Bloom's Taxonomy.

\section{CONLUSION AND FUTURE DEVELOPMENTS}

This paper presents a new integrated approach to developing the advanced education and training courses on Cloud Computing technologies that can be profiled both for full time university students, online education programs, and for acting IT professionals. The paper demonstrated the variety of approaches and methods used to improve the effectiveness of education on such complex technical domain as Cloud Computing in different education environments and for different groups of learners and trainees such as Bloom's Taxonomy and Andragogy.

As one of ongoing implementations of the proposed principles and methodologies, the authors are currently developing curriculum on Cloud Computing in conjunction with the IEEE eLearning Library program that provides wide spectrum of online educational materials for IEEE members. Once delivered, we look to report back to the community on the real-world results of these approaches.
When successfully tested, the course materials and obtained experience can be re-used for new instructional materials developments such as emerging Big Data and Data Intensive Science and Technologies such as falls into the Research Data Alliance (RDA) Interest Group on Educations and Skills Development scope [17]. However, similarly to the presented Cloud Computing curriculum, a new course must be supported with a well-defined Common Knowledge Base of the target professional education domain and corresponding architecture framework.

\section{REFERENCES}

[1] NIST SP 800-145, "A NIST definition of cloud computing”, [online] http://csrc.nist.gov/publications/nistpubs/800-145/SP800-145.pdf

[2] NIST SP 500-292, Cloud Computing Reference Architecture, v1.0. [Online] http://collaborate.nist.gov/twiki-cloud-computing/pub/ CloudComputing/ReferenceArchitectureTaxonomy/NIST_SP_500292_-_090611.pdf

[3] Demchenko, Yuri, David Bernstein, Adam Belloum, Ana Oprescu, Tomasz W. Wlodarczyk, Cees de Laat, New Instructional Models for Building Effective Curricula on Cloud Computing Technologies and Engineering. Proc. The 5th IEEE Internat ional Conference and Workshops on Cloud Computing Technology and Science (CloudCom2013), 2-5 December 2013, Bristol, UK.

[4] FG Cloud Technical Report (Part 1 to 7). [online] http://www.itu.int/en/ITU-T/focusgroups/cloud/Documents/FG-coudtechnical-report.zip

[5] Demchenko, Y., et al, Intercloud Architecture Framework for Heterogeneous Cloud based Infrastructure Services Provisioning OnDemand. The 2nd Intl Workshop on inter-Clouds and Collective Intelligence (iCCI-2013). The 27th IEEE International Conference on Advanced Information Networking and Applications (AINA2013). 2528 March 2013.

[6] Bernstein, D., Ludvigson, E., Sankar, K., Diamond, S., Morrow, M., Blueprint for the Intercloud - Protocols and Formats for Cloud Computing Interoperability. In Internet and Web Applications and Services, 2009. ICIW '09. Fourth International Conference on, 24-28 May 2009, Venice, Italy.

[7] Y.Demchenko, C. Lee, C.Ngo, C. de Laat, Federated Access Control in Heterogeneous Intercloud Environment: Basic Models and Architecture Patterns. IEEE Third Int. Workshop on Cloud Computing Interclouds, Multiclouds, Federations, and Interoperability (Intercloud2014), In Proc IEEE Int Conference on Cloud Engineering (IC2E), March 11, 2014, Boston, USA

[8] Anderson, L.W. (Ed.), A taxonomy for learning, teaching, and assessing: A revision of Bloom's Taxonomy of Educational Objectives (Complete edition). New York: Longman, 2001

[9] Bloom's taxonomy: the 21st century version. [online] http://www.educatorstechnology.com/2011/09/blooms-taxonomy21st-century-version.html

[10] Learning Domains or Bloom's Taxonomy, by Don Clark. [online] http://www.nwlink.com/ donclark/hrd/bloom.html

[11] Knowles, M.S., The Modern Practice of Adult Education: From Pedagogy to Andragogy, March 1988, ISBN-10: 0842822135

[12] Delahaye, B.L., D.C. Limerick, G. Hearn, The relationship between andragogical and pedagogical orientations and the implications for adult learning - Adult Education Quarterly, 44(4): 187-200. Sage Publications, 1994

[13] Merriam, S.B., Andragogy and Self-Directed Learning: Pillars of Adult Learning Theory

[14] Computer Science: Web Services and Cloud-Based Systems [online] http://gss.uva.nl/future-msc-students/informationsciences/information-sciences/information-sciences/content/folder3/computer-science.html

[15] Laureate Online Education. [online] http://www.university-liverpoolonline.com/online-learning/overview

[16] Laureate Online Education: Online Degree: MSc in Web Sciences and Big Data [online] http://www.university-liverpoolonline.com/programmes/informationtechnology/msc-in-websciences-and-big-data

[17] RDA Interest Group on Education and Training on handling of research data [online] https://www.rd-alliance.org/group/education-andtraining-research-data.html 